\title{
An Environmental Selection and Transfer Learning Based Dynamic Multiobjective Optimization Evolutionary Algorithm
}

Qiang He ( $\sim$ bchqiangu@126.com )

Xidian University

\section{Zheng Xiang}

Xidian University

Peng Ren

Xidian University

\section{Research Article}

Keywords: Artificial intelligence, Dynamic multiobjective optimization problems (DMOPs), evolutionary algorithms (EAs), environmental selection, transfer learning

Posted Date: March 26th, 2021

DOI: https://doi.org/10.21203/rs.3.rs-320752/v1

License: (9) This work is licensed under a Creative Commons Attribution 4.0 International License. Read Full License

Version of Record: A version of this preprint was published at Nonlinear Dynamics on January 12th, 2022. See the published version at https://doi.org/10.1007/s11071-021-07180-x. 


\title{
An Environmental Selection and Transfer Learning Based Dynamic Multiobjective Optimization Evolutionary Algorithm
}

\author{
Qiang He ${ }^{\dagger 1}$, Zheng Xiang ${ }^{\dagger}$, Peng Ren ${ }^{\dagger}$ \\ $\dagger$ The author is with school of telecommunications engineering, Xidian University, Xi'an 710071, China. \\ ${ }^{1}$ Correspondence: bchqiangu@126.com.
}

Received: $\quad$ Accepted:

\begin{abstract}
In recent years, the dynamic multiobjective optimization problems (DMOPs), whose major strategy is to track the varying PS (Pareto Optimal Solution, PS) and/or PF (Pareto Optimal Frontier), caused a great deal of attention worldwide. As a promising solution, reusing of "experiences" to establish a prediction model is proved to be very useful and widely used in practice. However, most existing methods overlook the importance of environmental selection in the evolutionary processes. In this paper, we propose a dynamic multiobjective optimal evolutionary algorithm which is based on environmental selection and transfer learning (DMOEA-ESTL). This approach makes full use of the environmental selection and transfer learning technique to generate individuals for a new environment by reusing experience to maintain the diversity of the population and speed up the evolutionary process. As experimental validation, we embed this new scheme in the NSGA-II (non-dominated sorting genetic algorithm). We test the proposed algorithm with the help of six benchmark functions as well as compare it with the other two prediction based strategies FPS (Forward-looking Prediction Strategy, FPS) and PPS (Population Prediction Strategy, PPS). The experimental results testify that the proposed strategy can deal with the DMOPs effectively.
\end{abstract}

Key words Artificial intelligence, Dynamic multiobjective optimization problems (DMOPs), evolutionary algorithms (EAs), environmental selection, transfer learning.

\section{Introduction}

A multiobjective optimization problem (MOP), which means an optimization problem with multiple objectives, is a major and widespread problem in both industrial applications and scientific research. Because of the changing of the objective functions and/or constraints, we call these problems as dynamic multiobjective optimization problems (DMOPs). Moreover, the change of 
PSs in decision space and/or the PFs in objective space are unpredictable or not observable, which makes the optimization problems more complex.

In recent years, there are much literature on DMOPs. These literature show that it is widely used in engineering and research on DMOPs in various application areas [1-10]. Meanwhile, some other scientific problems, such as intelligent learning [11], bilevel optimization [12], can be solved by means of DMOPs. DMOPs have been one of the hottest research topics, and many advanced control algorithms have been proposed in the late years. As so far, many new strategies have been proposed to tackle DMOPs, such as initialization, reference points, Kalman filter, dynamic learning et al. All these new methods are static methods based. However, various new theoretical and practical have proved that traditional optimization strategies may be hard to solve these complex problems efficiently.

Recently, evolutionary algorithms (EAs) are more and more popular in dealing with DMOPs partly because of the successful application of EAs in dealing with static MOPs. EAs, which were proposed by L. J. Fogel in the 1960's [13], have been widespread concerned in the last few years as they can perform the global search without differentiability of the objective functions. In addition, EAs are very efficient in dealing with DMOPs [14-16], such as video surveillance systems, control systems [17-20], design, and so on. The algorithms combined with EAs are named dynamic multiobjective optimization evolutionary algorithms (DMOEAs). As so far, most of the current DMOEAs are in their early stage of development.

As we know, the environment plays three different functions in the evolutionary process, and they are constraint, facilitating, and guiding. In one word, the main function of the environment is to guide the population to evolve in a better direction. At the same time, the evolutionary population also affects the evolutionary environment, such as the changes of the current evolutionary state, the update of environmental knowledge, etc. In the process of dynamic evolution, how to achieve the diversity and distribution of the population is the key to deal with the DMOPs. So, the method how to help the population to be reinitialized according to the new environment and enhance population diversity is important.

Significantly, the prediction based strategy performs very well in dealing with DMOPs. The cardinal idea of these methods is following the tracks and reusing of the good solutions when the environmental change occurs. It was proven theoretically and practically that this method was effective to deal with the DMOPs especially for those problems that the solutions obey an identical distribution.

The field application of traditional machine leaning already confirmed that it has a poor performance to predict the data when the data that used to train and the predicted data are not conform to the independent identical distribution. In recent years, a new prediction strategy based on transfer leaning [21] was proposed. In transfer learning strategy, the distribution of data that used in training and testing are different. However, prediction performance has been greatly improved by this strategy, and this method has been more and more popular. Therefore, the transfer learning, as an effective method in dealing with these complex problems, has played a significant role in solving of DMOPs.

In this paper, we propose a new strategy entitled DOMEA-ESTL, which integrates the environmental selection and transfer leaning schemes. Our motivation is to deal with the DMOPs more effectively. Firstly, we introduce the environmental selection into this system, which can help to get some guide-individuals by the environmental evaluate mechanism, and then divide individuals into two parts (with change and without change) according to the environmental regulation. Secondly, we predict the guide-individuals in a new environment for the optimization function according to the individual characteristics (predict the individual in a new environment by transfer learning for individuals with change or use the individual as the new guide-individuals in a new environment after correction for individuals without change). Thirdly, we get the optimal population in a new environment by the guide-individuals generated in the second step with NSGA-II scheme. Experimental results show that this 
new strategy can effectively solve DMOPs. Compared with traditional method, this new scheme can not only enhance population diversity, and make the population respond quickly to the different degrees of environmental changes, but also can accelerate the convergence of population by "experiences".

The contributions of this paper are summarized as follows:

(1) We consider an environmental selection strategy which can not only maintain population diversity but also accelerate the convergent speed. The environment, who plays a role of constraint, facilitating and guiding in the evolution process of the population, can help to establish a dynamic environment evolutionary model. Therefore, the environmental selection strategy improves the performance by guided fashion and it makes the population can respond quickly to the environmental changes. So, in this paper we take advantage of the environmental selection strategy to guide the evolution of population in a new environment.

(2) We introduce the transfer learning into this strategy. This new scheme make full use of the "experiences", which is also used in memory based method, and it significantly improve the search efficiency in solving the DMOPs.

This paper is organized as follows. Section II presents some preliminary studies and related works. Section III proposes the strategy based on environmental selection and transfer learning in detail. Test instances and performance metrics will be given in section IV. In section IV, experimental results are also presented and analyzed. Finally, Section V sums up some conclusions and gives some suggestions as the future research topics.

\section{Preliminary Studies and Research}

In this section, some basic theories used in this paper are introduced. The purpose is to introduce some basic knowledge and give certain background for readers to understand the sequel.

\subsection{Dynamic Multiobjective Optimization}

According to the characteristics of the nondeterminacy in dynamic multiobjective optimization problems, it can be divided into different categories [22-23]. Here, we focus on DMOPs that can be described as formula (1).

$$
\left\{\begin{array}{l}
\text { minimize } F(x, t)=\left(f_{1}(x, t), f_{2}(x, t), \ldots \ldots, f_{m}(x, t)\right)^{T} \\
\text { subject to } x \in \prod_{i=1}^{n}\left[a_{i}, b_{i}\right]
\end{array}\right.
$$

Where $t \in N$ represents time instants, $m$ is the number of objective functions, $x=\left(x_{1}, x_{2}, \ldots \ldots, x_{n}\right)^{T}$ is the decision vector, $-\infty<a_{i}<b_{i}<+\infty$ for all $i=1,2, \ldots, n, \prod_{i=1}^{n}\left[a_{i}, b_{i}\right] \subset R^{\wedge} n$ gives the interval ranges of the decision space. The objective vector $F(x, t): R^{\wedge} n \times T \rightarrow R^{\wedge} m$ consists of $m$ time varying objective functions: $f_{i}(x, t), \quad i=1, \ldots, m . R^{\wedge} m$ is the objective space. In the DMOP, there is an optimal trade off set which defines the optimality of a DMOP, and we call it PS and PF, respectively. The basic characteristics of a DMOP is that the PS and/or PF of it usually change over time.

Definition 1: Pareto Optimal Solution (PS). It is assumed that $x$ is the decision variable, $\Omega$ is the objective space, and $F$ is the objective function. Then, the PS which is the set of all nondominated solutions, can be denoted by formula (2).

$$
P S:=\left\{x \in \Omega \mid \nexists x^{*} \in \Omega, F\left(x^{*}\right) \prec F(x)\right\}
$$

Definition 2: Pareto Optimal Frontier (PF). It is assumed that $x$ is the decision variable, $F$ is the objective function. The $\mathrm{PF}$, which is the set of nondominated solutions with respect to the objective space, can be denoted by formula (3). 


$$
P F:=\{y=F(x) \mid x \in P S\}
$$

\subsection{Dynamic Multiobjective Optimization Evolutionary Algorithms (DMOEAs)}

It is important that a search algorithm can quickly adapt to environmental changes in dealing with DMOPs. Therefore, the population convergence and diversity is the key issue, which is also the central research hotspots and directions of designing EAs for solving DMOPs [22]. DMOEAs can solve the problem mentioned above successfully.

Detection and reflection of environmental change, optimization calculation are considered as three major steps of most existing DMOEAs. Algorithm 1 gives a general framework of a DMOEA [23-26]. Then, we will focus on a few major technologies used in the framework of a DMOEA.

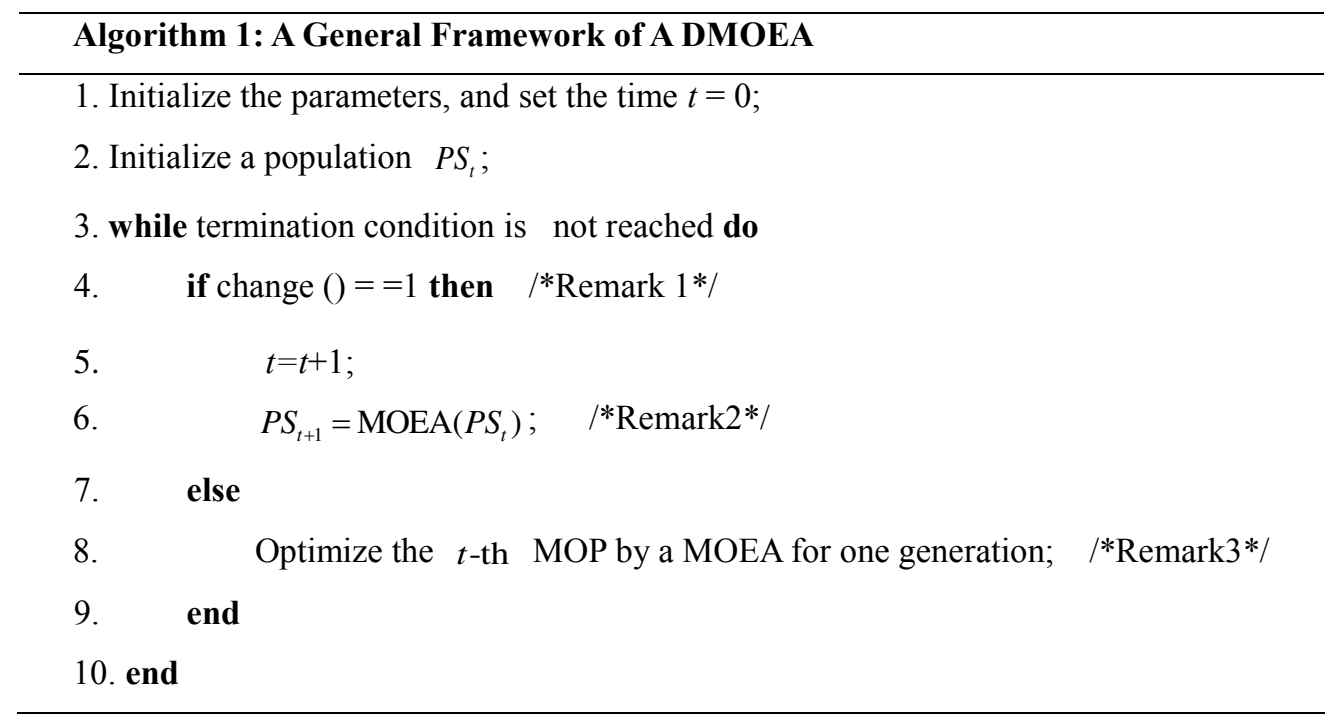

Remark 1: Change detection (line 4)

A change point $t_{c p}$ is defined as A, A. Z., B, B. Y. Q., C, H. L., B, S. Z. Z., B, P. N. S., \& D, Q. Z. . (2011):

$$
F\left(x, t_{c p}\right) \neq F\left(x, t_{c p}+1\right)
$$

where $F\left(x, t_{c p}\right)$ and $F\left(x, t_{c p}+1\right)$ are the objective functions at the time $t_{c p}$ and $t_{c p}+1$, respectively. With this step, a change can be detected when it occurs.

Remark 2: Change Reaction (Lines 5 and 6)

In response to the change in the environment, the system will make some corresponding response, which can be found in this step. There are many actions described in [27-37].

Remark 3: Optimization Calculation (Line 8)

This step is used to optimize the current MOPs for one generation. A multiobjective optimization evolutionary algorithm (MOEA) is usually applied to solve stationary MOPs. The purpose of the using of MOEA directly or with some modifications is to enhance the diversity of the population [38-45].

\subsection{Environmental Selection Mechanism}

In this paper, the environmental selection mechanism means that the environment can guide and promote the evolution by the 
functions of constraint, facilitating and guiding when an environmental change occurs. Constraint is the way to select the legitimacy of individuals in a new environment. Facilitating is used to promote the effectiveness of the evolution. Guiding can help to promote the distribution performance of the population. Three environmental elements are used to actualize the functions listing above, which are 1) environmental knowledge, 2) environmental evaluation, and 3) environmental regulation in a new environment.

First of all, the environmental knowledge is the environmental attribute in the process of evolution. There are two kinds of environmental knowledge which are static and dynamic, respectively. Static knowledge, which is constant values all over the process of evolution, consisted of environmental capacity and many other constant values. Dynamic knowledge, which is variable values, consisted of the direction of the evolution and the individuals obtained in the new environment etc.

Then, environmental evaluation mechanism, which is used to evaluate the living environment of population or individuals which contain individual location in the environment, the entire population distribution, etc. Environmental evaluation is used for guiding evolution.

Thirdly, environmental regulation means that individuals need to make some change in a new environment as well as the environment change.

With these three environmental elements mentioned above, it can guide and promote the evolution by the functions of constraint, facilitating and guiding when an environmental change occurs. Above all, the environmental selection mechanism can help to evolve to the desired direction according to the new environment.

\section{Environmental Selection and Transfer Learning Based Dynamic Multiobjective Optimization Evolutionary Algorithm}

In this section, we follow with interest how to treat with the change of environment by the proposed algorithm when a change is detected. In this section, a dynamic multiobjective optimization evolutionary algorithm based on environmental selection and transfer learning (DMOEA-ESTL) is proposed.

The solutions of a dynamic optimization problem always change with the environment, and the probability distributions also different from each other. Even though these distributions are not identical, but are correlated. It is assumed that if we transfer these individuals into a new space, in which the individuals are as "similar" as possible, we can construct an initial population by these available solutions, such that we can get the solutions for a new environment with low computational cost. In view of the above, we refers to transfer learning, which can deal with the problems in different but related fields, to improve the DMOEAs.

As mentioned above, because of the different probability distributions of the solutions of a DMOP, an invalid or even negative transfer learning will be leaded to if we transfer the characteristics of the individuals in a dynamic environment directly. In this paper, we consider an environmental selection strategy before the transfer learning, which is used to get the environment-adapted individuals to transfer learning.

The basic principle of the DMOEA-ESTL strategy is shown in figure 1.

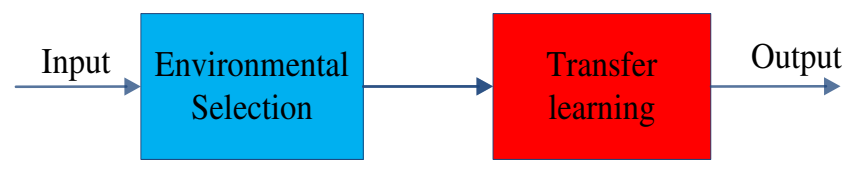

Figure 1. Principle of DMOEA-ESTL Strategy 


\subsection{Environmental Selection}

Traditional dynamic multiobjective optimization algorithm can track the new PSs/PFs quickly, but it ignores the role of dynamic environment in population evolution and lacks guidance search. From the perspective of evolution, "environment" is the sum of external conditions that an individual lives in. In this paper, the environment can be seen as a group of entities that can guide and promote the evolution of the population. In this paper, there are two reasons for using of the environmental selection strategy. Firstly, this strategy guides the search of population in a new environment by the information generated by the population in two different environments before and after the environmental change. Secondly, in order to avoid invalid or even negative transfer learning, we can select individuals with change as the source domain of the transfer learning.

We can store the population in dynamic environment by an idea which concur with the grid environment domain [46]. In this paper, we call the whole simulated environment as environment domain which consists of many same grids that called the unit domain. The grid environment domain records the convergence and distribution information generated during population evolution jointly. The individuals in the environment domain and unit domain is shown in figure 2.

In this paper, the dynamic environmental selection mechanism save the information of the grid environment domain by environmental knowledge firstly. The location and the distribution of individuals in figure 2 will change as well as the environment. Then it can evaluate the population with the environmental evaluation mechanism, which can calculate the difference of the unit domain before and after the environmental change. After that, we can get a serials of guide-individuals by these individuals with good evaluation results. These guide-individuals can be defined as:

$$
\begin{aligned}
& \text { gis }_{k}^{t}=x_{k}^{t}+\left|W_{k}^{t}-W_{k}^{t-1} G\right|, \text { if } W_{k}^{t}-W_{k}^{t-1}>0 \\
& g i s_{k}^{t}=x_{k}^{t}-\left|W_{k}^{t}-W_{k}^{t-1} G\right|, \text { if } W_{k}^{t}-W_{k}^{t-1}<0
\end{aligned}
$$

where $\operatorname{gis}_{k}^{t}$ is the guide-individual, $x_{k}^{t}$ denotes the individuals obtained at time $t . k=1,2, \ldots \ldots, n, n$ is the dimensions of the decision space. $G$ is a random number with a mean value of 0 and a variance of $1 . W_{k}^{t}$ is the center of non-dominated solutions obtained at time $t$.

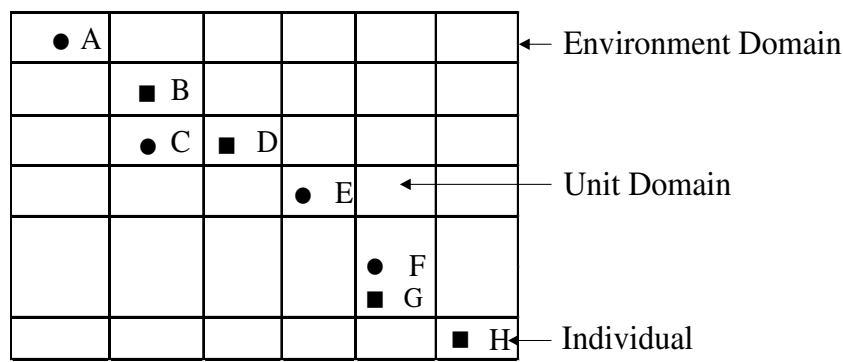

Figure 2. Individuals in Environment Domain and Unit Domain

For a changed environment, there may be different regulations. So the population should make the corresponding change. According to [46], in order to make the corresponding change for different individuals, the population in an environment was divided into three parts which is shown in figure 3. Then, in this paper, we consider two subpopulations which are subpopulation1 (with change) and subpopulation2 (without change). The individuals of subpopulation2 (without change) are non-dominated individuals who have the largest crowding-distance. The rest individuals of the population belong to subpopulation2. With the subpopulation1 (with change), we can get the guide-individuals in a new environment by the transfer learning strategy. With the subpopulation2 (without change), we can get the guide-individuals in a new environment corrected 
by (5).

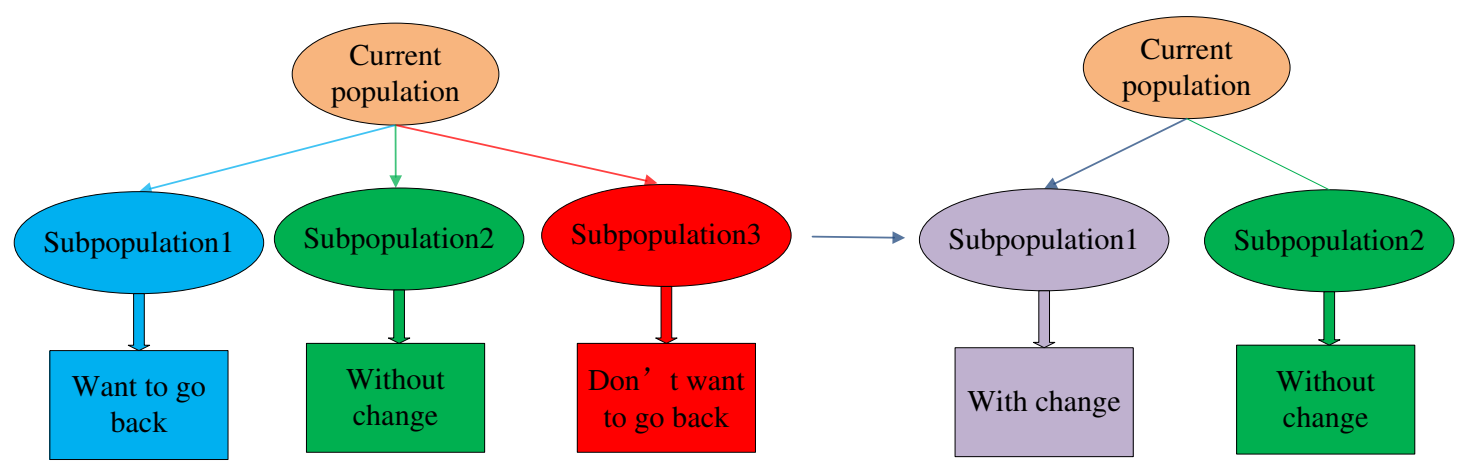

Figure 3. Division of Population

\subsection{Transfer Learning}

In this section, we adopt a new machine learning method, which is called transfer learning (TL). In principle, TL is a new method that memory based which can reuse the knowledge that we have already obtained. So, by this model, it can generate individuals for the optimization problem at a new time by the gained knowledge of finding PSs. Based on this strategy, the solutions of a DMOP can be obtained, and the process will be efficiently and effectively. For this new strategy, it is important to determine the transfer component.

\section{Transfer Component Analysis (TCA)}

TCA is a dimension reduction based method that can transform the problem of learning an entire kernel matrix to a low rank matrix $W$ instead. Then, the rest of this part will introduce how to get the matrix $W$ by TCA.

It is assumed that $X=\left\{x_{1}, x_{2}, \ldots, x_{n}\right\}$ and $Y=\left\{y_{1}, y_{2}, \ldots, y_{n}\right\}$ are sets of random variables whose probability distributions are $P$ and $Q$ on a domain $\chi$, respectively. So far, there are many criteria to measure the difference between different distributions, which is related to, but distinct from each other. Gretton, Smola and their other partners proposed a nonparametric distance estimation scheme called maximum mean discrepancy (MMD) to distinguish distributions in the reproducing kernel Hilbert space (RKHS) [47-48]. According to [47-49], the MMD is defined as:

$$
\operatorname{Dist}(X, Y)=\left\|\frac{1}{n_{1}} \sum_{i=1}^{n_{1}} \phi\left(x_{i}\right)-\frac{1}{n_{2}} \sum_{i=1}^{n_{2}} \phi\left(y_{i}\right)\right\|_{H}^{2}
$$

where $H$ is the RKHS. $\phi(\mathrm{x}): \chi->H$. By the so-called "kernel trick", we can rewrite (6) as formula (7).

$$
\begin{gathered}
\operatorname{Dist}\left(X_{s}^{\prime}, X_{T}^{\prime}\right)=\frac{1}{n_{1}^{2}} \sum_{i=1}^{n_{1}} \sum_{j=1}^{n_{1}} k\left(x_{S_{i}}, x_{s_{j}}\right)+\frac{1}{n_{2}^{2}} \sum_{i=1}^{n_{2}} \sum_{j=1}^{n_{2}} k\left(x_{T_{i}}, x_{T_{j}}\right) \\
-\frac{1}{n_{1} n_{2}} \sum_{i=1}^{n_{1}} \sum_{j=1}^{n_{2}} k\left(x_{S_{i}}, x_{T_{j}}\right) \\
=\operatorname{tr}(K L) \\
K=\left[\begin{array}{ll}
K_{S, S} & K_{S, T} \\
K_{T, S} & K_{T, T}
\end{array}\right]
\end{gathered}
$$

where $X_{S}^{\prime}=\left\{x_{S_{i}}^{\prime}\right\}=\left\{\phi\left(x_{T_{i}}\right)\right\}, X_{T}^{\prime}=\left\{x_{T_{i}}^{\prime}\right\}=\left\{\phi\left(x_{T_{i}}\right)\right\}$ are the source domain and target domain, respectively. $K_{X, Y}$ is a 
kernel matrix with $k_{i, j}=\kappa\left(x_{i}, y_{i}\right)=\phi\left(x_{i}\right)^{T} \phi\left(y_{i}\right)$. By the so-called "empirical kernel map", we can rewrite (7) as formula (10).

$$
\begin{gathered}
L=\left[L_{i j}\right]=\left\{\begin{array}{cc}
\frac{1}{n_{1}^{2}} & x_{i}, x_{j} \in X \\
\frac{1}{n_{2}^{2}} & x_{i}, x_{j} \in Y \\
-\frac{1}{n_{1} n_{2}} & \text { otherwise }
\end{array}\right. \\
\operatorname{Dist}\left(X_{s}^{\prime}, X_{T}^{\prime}\right)=\operatorname{tr}\left(\left(K W W^{T} K\right) L\right) \\
=\operatorname{tr}\left(W^{T} K L K W\right)
\end{gathered}
$$

When minimizing formula (10), it needs a regularization term $\operatorname{tr}\left(W^{T} W\right)$. Thus, the problem can be changed into formula (11).

$$
\begin{gathered}
\begin{cases}\min _{W} & \operatorname{tr}\left(W^{T} W\right)+\mu \cdot \operatorname{tr}\left(W^{T} K L K W\right) \\
\text { s.t. } & W^{T} K H K W=I\end{cases} \\
H=I_{n_{1}+n_{2}}-\frac{1}{n_{1}+n_{2}} P P^{T}
\end{gathered}
$$

Where $I$ is a $(m+n) \times(m+n)$ unit matrix. $W^{T} W$ is a regularization term. $P$ is a $(m+n) \times 1$ matrix whose elements are all one. $m$ and $n$ are the number of samples in the source and objective domains, respectively. $\mu$ is the trade off parameter.

\section{DMOEA-TL}

In this section, the pseudo-codes of DMOEA-TL algorithm, which is a transfer learning based scheme that can help to search for the PFs at a new time, is given. More specifically, we take the historical data of PFs that obtained at time $t$ that generated by transfer learning method as a source domain, the object solutions of the next time (time $t+1$ ), as the objective domain, and then construct a mapping function $\varphi($.) by the domain adaptation approach. This mapping function will embed the distributions that the source and objective domain obey separately into a latent space, and in that space the difference between the two distributions will be as small as possible.

The pseudo-code of DMOEA-TL algorithm is given in algorithm 2. According to the DMOEA-TL scheme, the PFs at a new time can be obtained.

\section{Algorithm 2: DMOEA-TL}

Input: The function to be optimized $F_{t+1}($.$) ; the Optimal solution of F_{t}($.$) at time t, P F_{t}=\left\{p_{l}, \ldots, p_{m}\right\}$, a kernel function $\kappa(.,$.$) .$

Output: A population Pop-reinit.

1. Initialization, parameter setting;

2. For $F_{t}($.$) and F_{t+1}($.$) , randomly set two sets of the solutions X_{S}$ and $Y_{T} ; / *$ Remark $1 * /$

3.Calculating of the values of $F_{t}\left(X_{S}\right)$ and $F_{t+1}\left(Y_{T}\right)$;

4. $W=T C A\left(\left\{F_{t}\left(X_{S}\right)\right\},\left\{F_{t+1}\left(Y_{T}\right)\right\}, \kappa\right)$; 


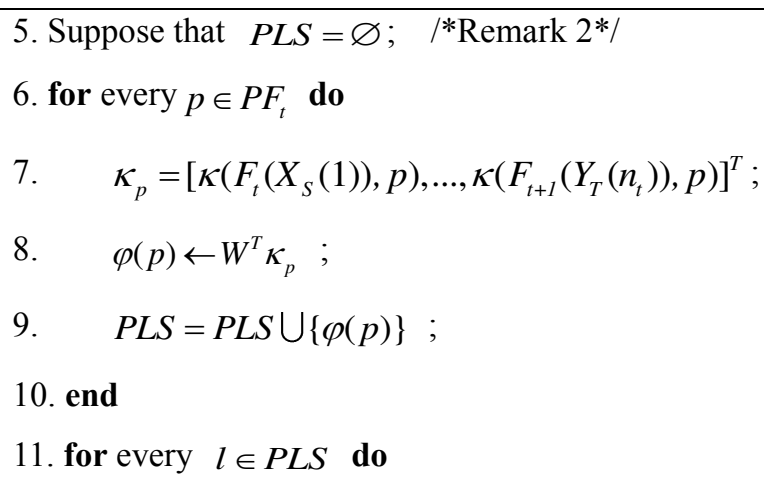

12. $x=\arg \min \left\|\varphi\left(F_{t+1}(x)\right)-j\right\| ; \quad / * \operatorname{Remark} 3 * /$

13. Pop-reini=Pop-reini $\bigcup\{x\}$;

14. end

15. return Pop-reini;

Remark 1: Generally speaking, the number of the components of $X_{S}$ and $Y_{t}$ are predefined. It is assumed that $\left|X_{S}\right|=n_{S}$ and $\left|Y_{t}\right|=n_{t}$. In general, the more number of sampling, the better prediction result but higher cost will be, so the number of solutions to be generated in this step usually depends on the available resources.

Remark 2: PLS is the abbreviation for particle in the latent space. It can be regarded as a set of the mapped solutions in the latent space.

Remark 3: In this step, our motivation is to get an individual $x$, such that the result $\varphi\left(F_{t+l}(x)\right)$ is as close as possible to $j \in P L S$ in the latent space. In this regard, it is worthy to first solve a single objective optimization problem, and the solutions can be used in dealing with DMOPs.

Figure 4 illustrates the key steps of the DMOEA-TL algorithm, which can help readers quickly catch the key thought of this strategy. What needs to be emphasized is that the input to the TCA are the samples of the solutions at time $t$ and $t+1$, and its output is a transformation matrix $W$, by which we can construct the latent space. Step 1, it depicts the method how to map the PF at time $t$ into the latent space, then steps 2 and 3 describe the process of searching for an initial population which can be used as the solution of the dynamic optimization problem at a new time of $t+1$. 


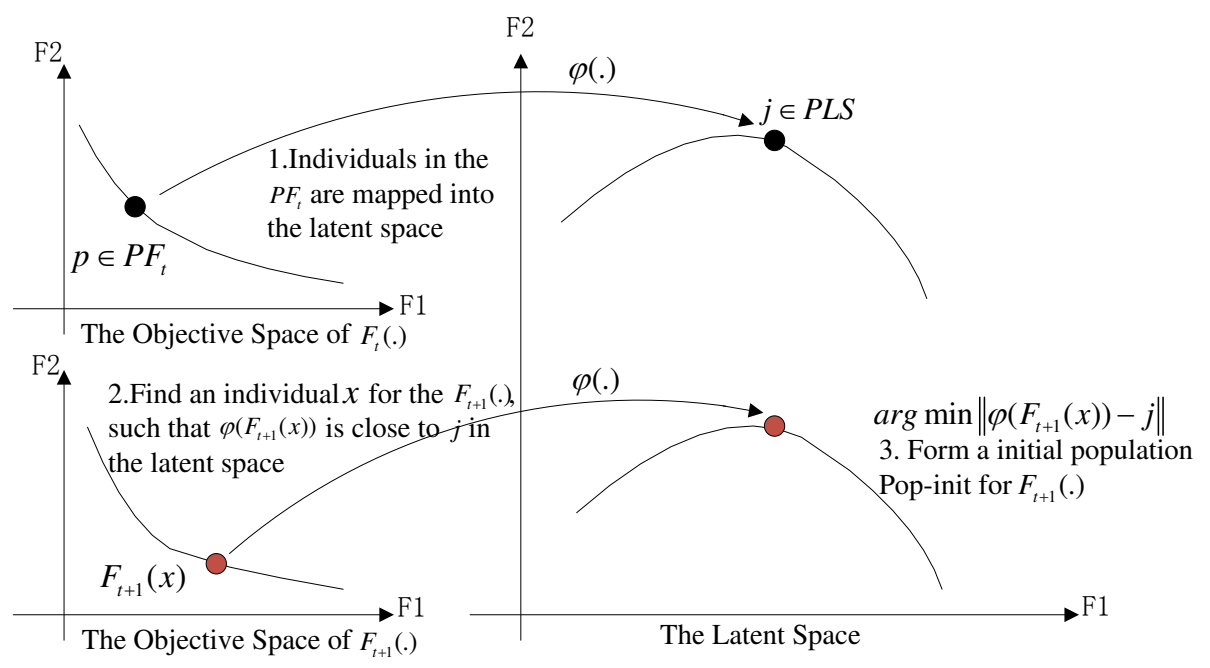

Figure 4. Key Steps of DMOEA-TL Algorithm. F1、F2 represent the objective function respectively.

\subsection{DMOEA-ESTL}

In this section, an environmental selection and transfer learning based DMOEA which is called DMOEA-ESTL will be given. Based on DMOEA-TL, the DMOEA-ESTL introduces the environmental selection mechanism before transfer learning. This method combines the advantages of environmental selection and transfer learning. Theoretically, the environmental selection scheme can guide the evolution of individuals by the function of constraint, facilitating and guiding, and the transfer learning makes full use of the "past experience" to get the new individuals when the change is detected. Therefore, the DMOEA-ESTL is a very effective strategy to solve the DMOPs. The principle of DMOEA-ESTL is shown in figure 5. As a case of study, we select a well represented algorithm NSGA-II to verify our approach. The pseudo-codes of DMOEA-ESTL is given in algorithm 3.

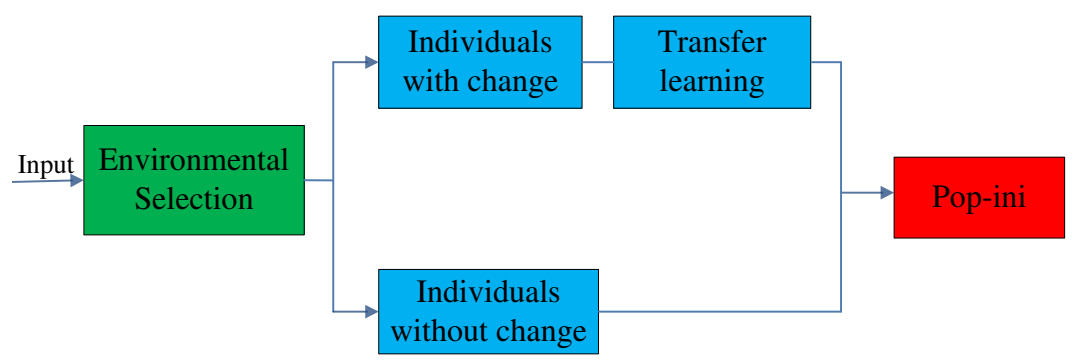

Figure 5. Principle of DMOEA-ESTL

\begin{tabular}{l} 
Algorithm 3: DMOEA-ESTL \\
\hline Input: The function $F(X)$, Current-Pop, total number of generation, frequency of change, a kernel function \\
$\kappa(.,$.$) etc.$ \\
Output: A new population Pop-reinit. \\
\hline 1. Initialization; \\
2. Detection of environmental changes; \\
3. if environmental change==1 \\
4. Evaluation of the individuals, and generating of guide-individuals1;
\end{tabular}


5. Divide the population into two parts;

6. For subpopulation1:

Transfer learning,

Generating of guide-individuals2 by formula (5) ;

6. For subpopulation 2:

7. Generating of guide-individuals 3 by formula (5);

8. Recombine, Current-Pop=guide-individuals $1 \cup$ guide-individuals $2 \cup$ guide-individuals3;

9. Pop-reinit=NSGA-II(Current-Pop);

10. else

11. Pop-reinit=NSGA-I(Current-Pop);

12. end

\section{Empirical Study}

\subsection{Test Instances}

The benchmark function is important for the test and design of the algorithm. There are many typical DMOPs in [14] and [50], and many variants of these problems in [26] and [51]. In this experiment, there are six standard functions, and all of these functions are selected from various DMOPs types [52] which can be used to verify the performance of DMOPs.

Table 1 Test instances used in this experiments

\begin{tabular}{|c|c|c|c|}
\hline Instance & Search Space & Objectives, PS and PF & Remarks \\
\hline DMOP1 & {$[0,1] \times[-1,1]^{n-1}$} & $\left\{\begin{array}{l}f_{1}(x, t)=x_{1}, f_{2}(x, t)=g * h \\
g=1+9 \sum_{i=2}^{n} x_{i}^{2}, h=1-\left(\frac{f_{1}}{g}\right)^{H} \\
H=1.25+0.75 \sin (0.5 \pi t), t=\left\lfloor\tau / \tau_{T}\right\rfloor / n_{T} \\
P S(t): 0 \leq x_{1} \leq 1, x_{i}=0, i=2, \ldots \ldots, n \\
P F(t): f_{2}=1-f_{1}^{H}, 0 \leq f_{1} \leq 1\end{array}\right.$ & $\begin{array}{l}\text { Two objectives } \\
\text { PF changes } \\
\text { PS is fixed }\end{array}$ \\
\hline DMOP2 & {$[0,1] \times[-1,1]^{n-1}$} & $\left\{\begin{array}{l}f_{1}(x, t)=x_{1}, f_{2}(x, t)=g * h, t=\left\lfloor\tau / \tau_{T}\right\rfloor / n_{T} \\
g=1+\sum_{i=2}^{n}\left(x_{i}-G\right)^{2}, h=1-\left(\frac{f_{1}}{g}\right)^{H} \\
G=\sin (0.5 \pi \mathrm{t}), H=1.25+0.75 \sin (0.5 \pi t) \\
P S(t): 0 \leq x_{1} \leq 1, x_{i}=G, i=2, \ldots \ldots, n \\
P F(t): f_{2}=1-f_{1}^{H}, 0 \leq f_{1} \leq 1\end{array}\right.$ & $\begin{array}{l}\text { Two objectives } \\
\text { PF changes } \\
\text { PS changes }\end{array}$ \\
\hline DMOP3 & {$[0,1] \times[-1,1]^{n-1}$} & $\begin{array}{l}f_{1}\left(x_{r}, t\right)=x_{r}, f_{2}\left(x \backslash x_{r}\right)=g * h, t=\left\lfloor\tau / \tau_{T}\right\rfloor / n_{T} \\
g=1+\sum_{i=1}^{x \backslash x_{r}}\left(x_{i}-G\right)^{2}, h=1-\sqrt{\frac{f_{1}}{g}} \\
G=\sin (0.5 \pi t), r=\bigcup(1,2, \ldots, n) \\
P S(t): 0 \leq x_{1} \leq 1, x_{i}=G, i=2, \ldots \ldots, n \\
P F(t): f_{2}=1-\sqrt{f}, 0 \leq f_{1} \leq 1\end{array}$ & $\begin{array}{l}\text { Two objectives } \\
\text { PF is fixed } \\
\text { PS changes }\end{array}$ \\
\hline
\end{tabular}




\begin{tabular}{|c|c|c|c|}
\hline FDA4 & {$[0,1]^{n}$} & $\left\{\begin{array}{l}f_{1}(x, t)=(1+g) \cos \left(0.5 \pi x_{2}\right) \cos \left(0.5 \pi x_{1}\right) \\
f_{2}(x, t)=(1+g) \cos \left(0.5 \pi x_{2}\right) \sin \left(0.5 \pi x_{1}\right) \\
f_{3}(x, t)=(1+g) \sin \left(0.5 \pi x_{2}\right), t=\left\lfloor\tau / \tau_{T}\right\rfloor / n_{T} \\
g(x, t)=\left|\sum_{i=3}^{n}\left(x_{i}-G\right)^{2}\right|, G=\sin \left(0.5 \pi \frac{t}{n_{T}}\right) \\
P S(t): 0 \leq x_{1}, x_{2} \leq 1, x_{i}=G, i=3, \ldots \ldots, n \\
P F(t): f_{1}=\cos (\mu) \cos (v), f_{2}=\cos (\mu) \sin (v), f_{3}=\sin (\mu), 0<\mu, v<\pi / 2\end{array}\right.$ & $\begin{array}{l}\text { Three objectives } \\
\text { PF is fixed } \\
\text { PS changes }\end{array}$ \\
\hline FDA6 & {$[0,5]^{n}$} & $\left\{\begin{array}{l}f_{1}(x, t)=\left|x_{1}-a\right|^{H}+\sum_{i \in I_{1}} y_{i}^{2}, a=2 \cos (1.5 \pi t) \sin (0.5 \pi t)+2 \\
f_{2}(x, t)=\left|x_{1}-a-1\right|^{H}+\sum_{i \in I_{2}} y_{i}^{2}, b=2 \cos (1.5 \pi t) \cos (0.5 \pi t)+2 \\
y_{i}=x_{i}-b-1+\left|x_{1}-a\right|^{H+\frac{i}{n}}, H=1.25+0.75 \sin (\pi t), t=\left\lfloor\tau / \tau_{T}\right\rfloor / n_{T}, i=2, \ldots \ldots ., n \\
I_{1}=\{i \mid 1 \leq i \leq n, i \text { is odd }\}, I_{2}=\{i \mid 1 \leq i \leq n, i \text { is even }\} \\
P S(t): a \leq x_{1} \leq a+1, x_{i}=b+1-\left|x_{1}-a\right|^{H+\frac{i}{n}} \\
P F(t): f_{2}=(1-S)^{H}, f_{1}=S^{H}, 0 \leq S \leq 1\end{array}\right.$ & $\begin{array}{l}\text { Two objectives } \\
\text { PF changes } \\
\text { PS changes }\end{array}$ \\
\hline FDA7 & {$[0,5]^{n}$} & $\left\{\begin{array}{l}f_{1}(x, t)=\left|x_{1}-a\right|^{H}+\sum_{i \in I_{1}} y_{i}^{2}, f_{2}(x, t)=\left|x_{1}-a-1\right|^{H}+\sum_{i \in I_{2}} y_{i}^{2} \\
y_{i}=x_{i}-b-1+\left|x_{1}-a\right|^{H+\frac{i}{n}}, H=1.25+0.75 \sin (\pi t) \\
a=1.7(1-\sin (\pi t))+3.4, b=1.4(1-\sin (\pi t)) \cos (\pi t)+2.1 \\
I_{1}=\{i \mid 1 \leq i \leq n, i \text { is odd }\}, I_{2}=\{i \mid 1 \leq i \leq n, i \text { is even }\}, t=\left\lfloor\tau / \tau_{T}\right\rfloor / n_{T} \\
P S(t): a \leq x_{1} \leq a+1, x_{i}=b+1-\left|x_{1}-a\right|^{H+\frac{i}{n}}, i=2, \ldots \ldots ., n \\
P F(t): f_{2}=(1-S)^{H}, f_{1}=S^{H}, 0 \leq S \leq 1\end{array}\right.$ & $\begin{array}{l}\text { Two objectives } \\
\text { PF changes } \\
\text { PS changes }\end{array}$ \\
\hline
\end{tabular}

These functions can be divided into two categories. The first one belong to DMOP functions, that are DMOP1 DMOP3, which are functions with linear correlation between the decision variables. The second one belong to FDA functions, that are FDA4, FDA6 and FDA7, which are three functions with linear correlation and two functions with nonlinear correlation between the decision variables, respectively. Table 1 gives all these six standard test functions and their PF and PS in details.

\subsection{Performance Indications for Dynamic Optimization}

In order to evaluate the performance of the new scheme, a modified version of the inverse generational distance, abbreviated as IGD, is used here. It is a widely used metric for evaluating the performance of DMOEAs [14-15] which can measure both convergence and distribution of the solution set obtained by a DMOEA. The IGD metric is defined by the formula (12).

$$
\operatorname{IGD}\left(R^{t^{*}}, R^{t}\right)=\frac{\sum_{v \in P^{*}} d\left(v, R^{t}\right)}{\left|R^{t^{*}}\right|}
$$

Where $R^{t^{*}}$ is a set that contains the solutions uniformly sampled from the true PF, and $R^{t}$ is the non-dominated solution set achieved by a DMOEA. $d\left(v, R^{t}\right)$ is the minimum distance from $v$ and a solution in $R^{t}$. The IGD metric is also called Dmetric. The ideal IGD value is zero, which means that it has obtained the best convergence and diversity.

One variant of the IGD, called MIGD, can also be used to evaluate dynamic multiobjective optimization algorithms [25], and it takes the mean of the IGD values in some time steps over a run as the performance metric, given by formula (13). 


$$
\operatorname{MIGD}\left(R^{t^{*}}, R^{t}\right)=\frac{1}{|T|} \sum_{t \in T} \operatorname{IGD}\left(R^{t^{*}}, R^{t}\right)
$$

where $T$ is a set of discrete time points in a run, and $|T|$ is the cardinality of $T$.

\subsection{Parameter Settings}

The performance of the algorithm must be proved by experiments, so two recently proposed prediction based methods, namely, the population prediction strategy (PPS) [26], and a forward-looking prediction strategy (FPS) [53], for dealing with DMOPs is compared with the proposed strategy DMOEA-ESTL in the experimental studies. For a fair comparison, all these three strategies are embedded into NSGA-II algorithm. The parameter settings for the three compared algorithms and test instances are presented in table 2 .

\subsection{Experimental Results and Analysis}

The PSs and PFs of the testing functions have different shapes and each function belongs to a certain type, figure 6 and figure 7 describe the true PS and PF of the six testing functions.

Table 2 Parameter settings

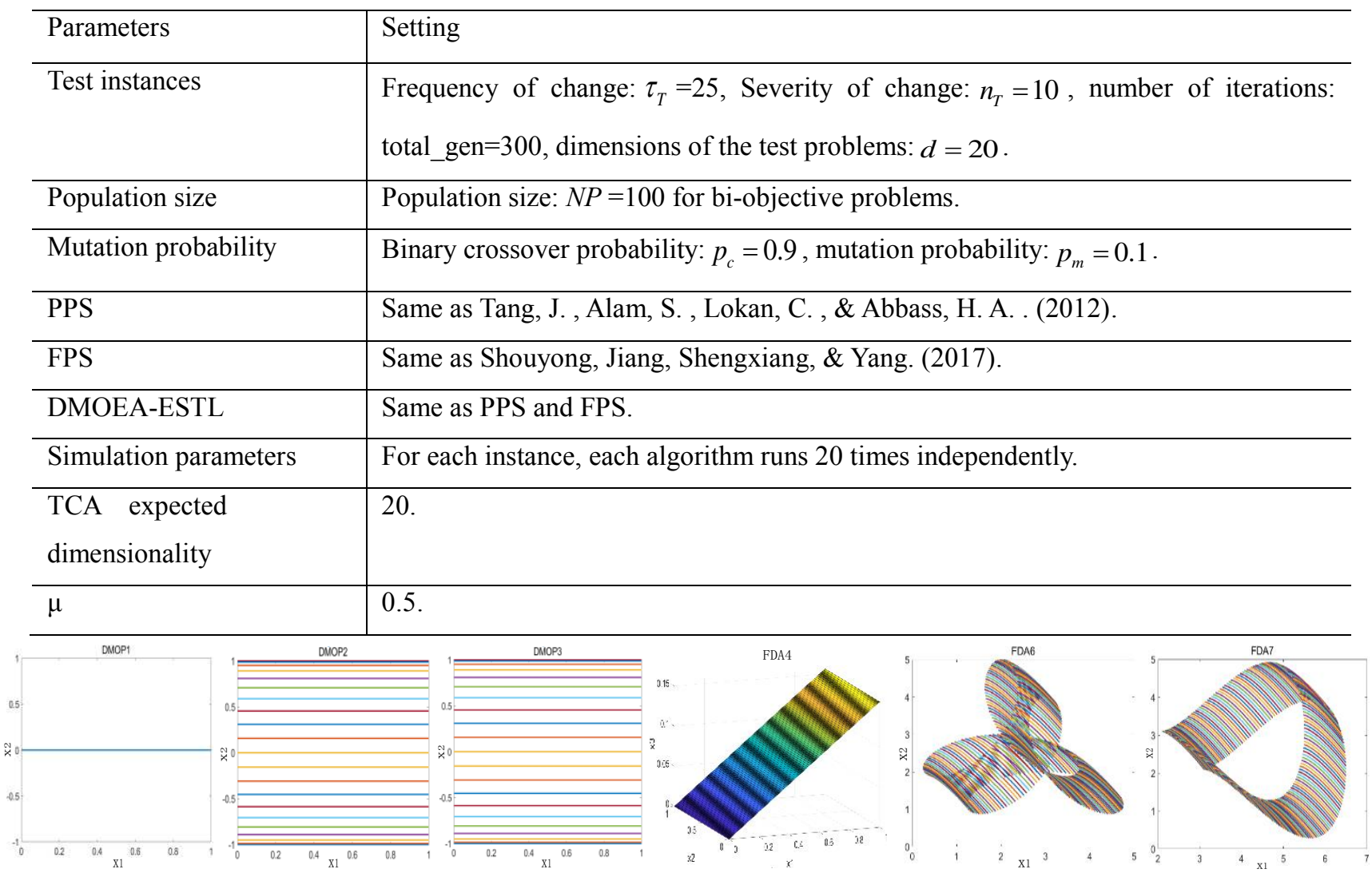

Figure 6. True PSs of the Six Testing Functions 

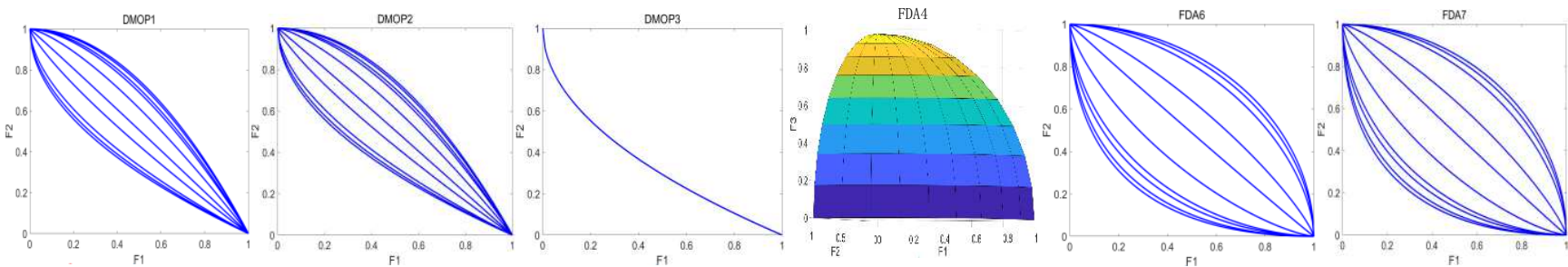

Figure 7. True PFs of the Six Testing Functions

Figure 8 gives the results of population obtained by FPS, PPS and DMOEA-ESTL at $\mathrm{t}=125,130,135,140,145,150$ among 20 runs on DMOP1. Then, Figure 9 gives the results of population obtained by FPS, PPS and DMOEA-ESTL at $\mathrm{t}=143,145$, 147, 149, 151, 153 among 20 runs on FDA6. These two figures show that the DMOEA-ESTL can approximate the PFs very well for these two functions, and the performance of the FPS and PPS is slightly worse than DMOEA-ESTL.
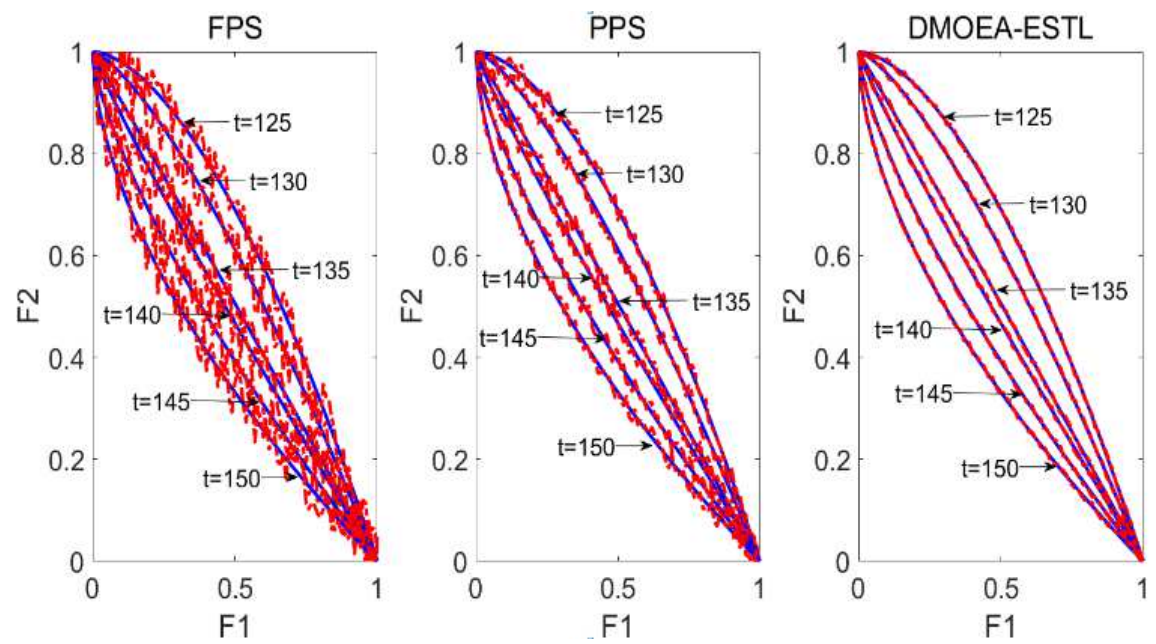

Figure 8. Populations Obtained by FPS, PPS, and DMOEA-ESTL on DMOP1
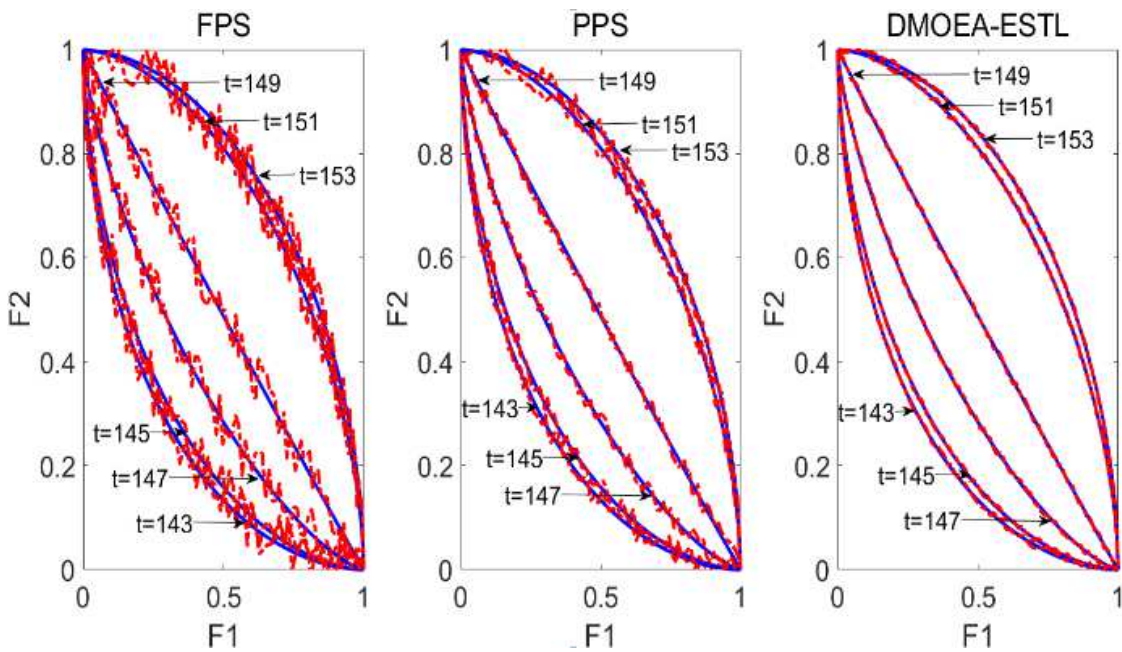

Figure 9. Populations Obtained by FPS, PPS, and DMOEA-ESTL on FDA6

Figure 10 shows the average MIGD of the three algorithms FPS, PPS, and DMOEA-ESTL on six test functions over 20 runs. In this simulation system, the environment changes every 30 generations, and a total of 10 environmental change is considered. Then, we give all these experimental results of the statistical average MIGD in table 3.

It must be emphasized that all of these results are obtained without tuning any one of the parameters, which means that 
the experimental conditions are the same. The main reason for not adjusting the parameters is that we can get a better performance by tuning any one of the parameters. So, we can get a fair result with the same parameters.

From these results, the following observations can be made:

1) For this initial phase, the performance of these three algorithms is relatively poor. The reason might be that

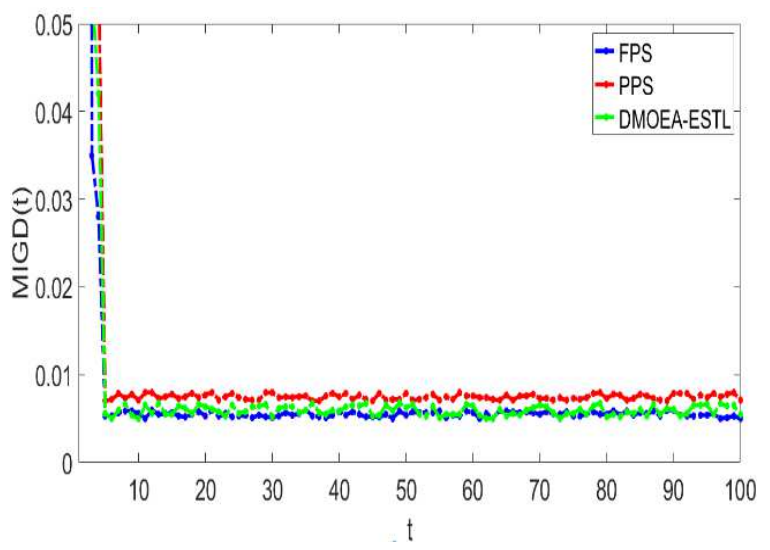

(a) DMOP1

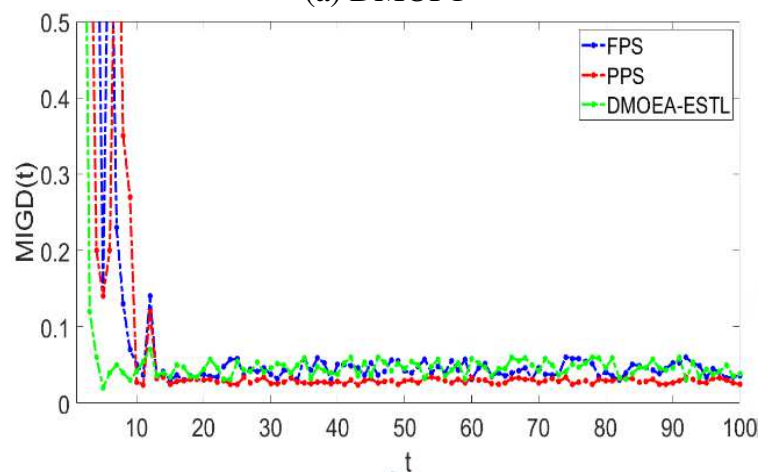

(b) DMOP2

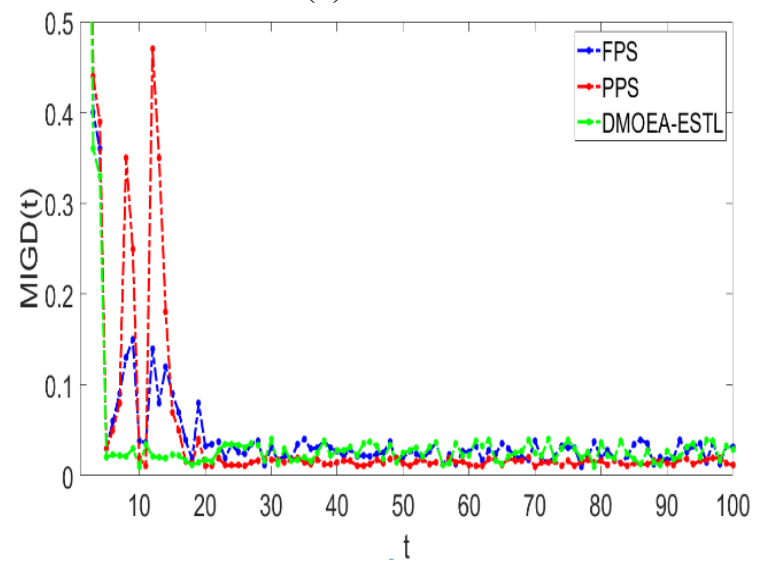

(c) DMOP3 


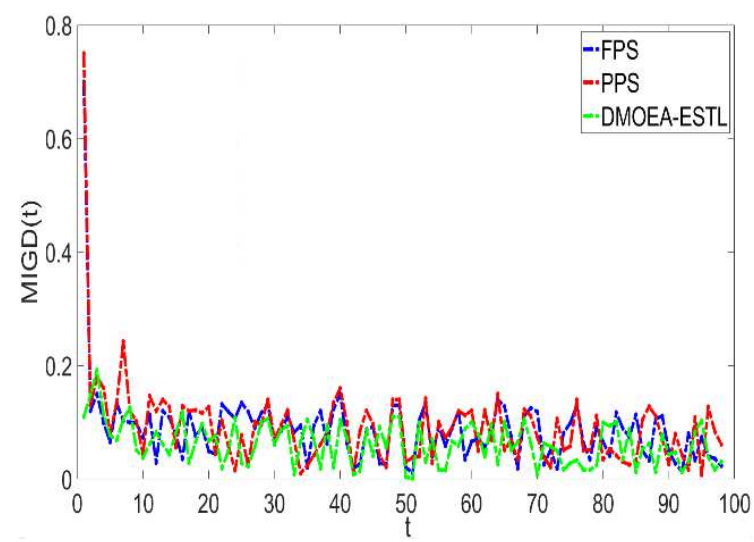

(d) FDA4

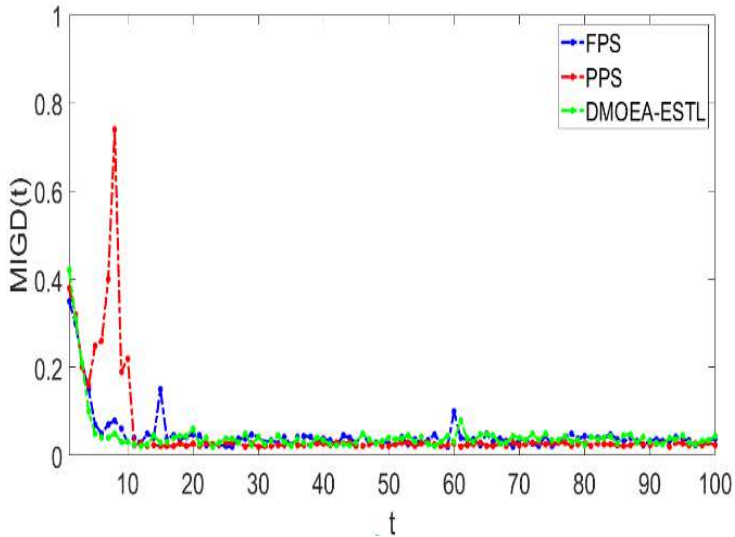

(e) FDA6

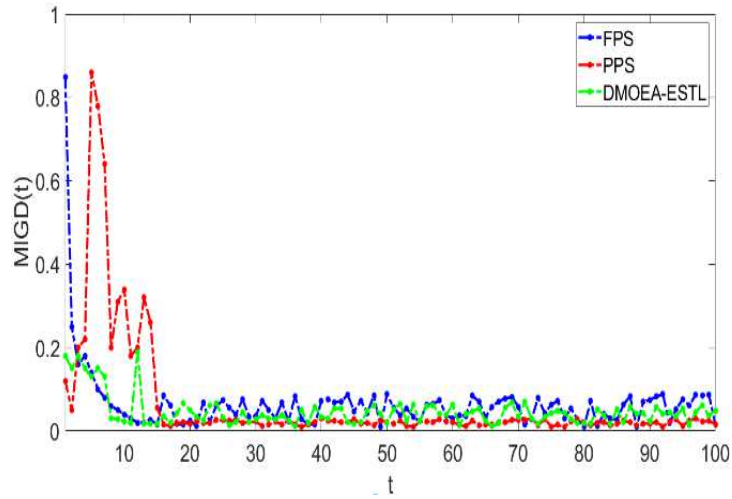

(f) FDA7

Figure 10. Average MIGD of Three Algorithms

the lacking of historical information at the beginning. So, when $1<t \leq 20$, the MIGD of these three algorithms is much larger than that of other time. When $t>20$ the performance of these three algorithms is gradually improved with the passage of time. The reason might be that there is sufficient data after evolutionary operation.

2) An obvious conclusion is that the average MIGD values of DMOEA-ESTL are less than PPS and FPS, especially in the range of $0<t \leq 20$. The observation means that DMOEA-ESTL can perform better performances when the environmental changes is detected.

3) As time goes on, the environment will change periodically, and the experience will be accumulated gradually. So, the convergence and diversity of PPS will gradually stabilized, and the reason is the combination of memory scheme. The performance of PPS will be much better than that of DMOEA-ESTL. In a word, the difference of these two strategies is small, 
and the performance of DMOEA-ESTL is better than that of FPS.

4) DMOP1 is the problem with a fixed PS. Theoretically, the diversity and convergence of the population are contradictory, and they can not reach the optimal at the same time. The performance is much better than that of other testing functions which indicates that the using of historical data can improve the performance of convergence.

5) For these three algorithms, the performance of DMOEA-ESTL scheme is not worse than that of the other two strategies, which is because that the DMOEA-ESTL takes advantage of the transfer learning which can achieve better learning results in the absence of source data to generate new individuals in a new environment.

6) As shown in figure 10, the convergence time of the DMOEA-ESTL strategy is the shortest for all these experiments. For example, the convergence time of DMOEA-ESTL on DMOP1 is close to $t=5$, and the convergence time of the other four testing functions are also in the range of $t<20$, which is shorter than that of the other two (FPS and PPS) strategies.

In summary, these experimental results show that the DMOEA-ESTL performs better than that of other two algorithms on the six testing functions. It also shows that the combination of environmental selection and transfer learning can improve the performance of the existing multiobjective EAs appreciably without significant modifications for solving the DMOPs. There we summed up the reason to the results. The environmental selection of DMOEA-ESTL divides the current population into two parts (with change and without change) according to the different behavioral characteristics of each individual when a change is detected. According to different environmental changes, these two subpopulations can be treated differently. Meanwhile, with the transfer learning, the subpopulation with change will be guided to evolve toward their desired environments according to the new environmental knowledge and regulation. Therefore, DMOEA-ESTL is able to respond more quickly to environmental change and can perform better than the other two algorithms.

Table 3 Statistical average MIGD of different algorithms

\begin{tabular}{llll}
\hline MIGD & FPS & PPS & DMOEA-ESTL \\
\hline DMOP1 & $5.12 \mathrm{E}-3$ & $7.56 \mathrm{E}-3$ & $5.04 \mathrm{E}-3$ \\
\hline DMOP2 & $5.16 \mathrm{E}-2$ & $5.46 \mathrm{E}-2$ & $3.31 \mathrm{E}-2$ \\
\hline DMOP3 & $3.02 \mathrm{E}-2$ & $3.68 \mathrm{E}-2$ & $2.98 \mathrm{E}-2$ \\
\hline FDA4 & $9.32 \mathrm{E}-2$ & $9.76 \mathrm{E}-2$ & $9.19 \mathrm{E}-2$ \\
\hline FDA6 & $3.58 \mathrm{E}-2$ & $3.87 \mathrm{E}-2$ & $3.22 \mathrm{E}-2$ \\
\hline FDA7 & $3.88 \mathrm{E}-2$ & $6.88 \mathrm{E}-2$ & $2.95 \mathrm{E}-2$ \\
\hline
\end{tabular}

\section{Conclustion and Future Work}

This paper proposes a new strategy which is called DMOEA-ESTL to track the changing PS and/or PF of dynamic multiobjective optimization problems. The main idea of this strategy is to take advantage of the information of population in a new environment and transfer the main characteristics to accelerate tracking capability for PS and/or PF. The main steps of the algorithm can be described as: Firstly, we introduce the environmental selection into this system, which can help to get some guide-individuals by the environmental evaluate mechanism, and then divide individuals into two parts (with change and without change) according to the environmental regulation. Secondly, we predict the guide-individuals in a new environment for the optimization function according to the individual characteristics (predict the individual in a new environment by transfer learning for individuals with change or use the individual as the new guide-individuals in a new environment after correction for individuals without change). Thirdly, we get the optimal population in a new environment by 
the guide-individuals generated in the second step with NSGA-II scheme. The DMOEA-ESTL is designed to construct the initial population in order to realize the balance between the convergence and diversity.

Experimental results show that the proposed DMOEA-ESTL strategy performs better than the other two prediction based strategies FPS and PPS. Although DMOEA-ESTL has been demonstrated can outperform than the other two strategies on problems with dramatic changes in PF and/or PS or with complex correlation relationship between the decision variables, but it is also expected to examine its performance against more other types of DMOEAs. Therefore, it is called an urgent need to design a strategy that can solve many different types of multiobjective optimization problems simultaneously.

\section{Author Contributions}

For Conceptualization, Qiang He and Peng Ren; methodology, all authors have contributed to the methodology presented in this article ; validation, Peng Ren and Zheng Xiang; formal analysis, Qiang He; investigation, Qiang He; resources, Zheng Xiang; writing, all authors have contributed to writing of this article.

\section{Funding}

This research received no external funding.

\section{Declarations}

The authors declare no conflict of interest.

\section{References}

[1] Jiao, R. , Zeng, S. , Li, C. , \& Pedrycz, W. .. Evolutionary Constrained Multi-objective Optimization using NSGA-II with Dynamic Constraint Handling. 2019 IEEE Congress on Evolutionary Computation (CEC). IEEE, (2019).

[2] Abello, M. B. , Bui, L. T. , \& Michalewicz, Z. .. An adaptive approach for solving dynamic scheduling with time-varying number of tasks - Part II. Evolutionary Computation (CEC), 2011 IEEE Congress on. IEEE, (2011).

[3] Ma, X. , Yang, J. , Sun, H. , Hu, Z. , \& Wei, L. .. Multiregional co-evolutionary algorithm for dynamic multiobjective optimization. Information Sciences, 545, (2020).

[4] Tang, J. , Alam, S. , Lokan, C. , \& Abbass, H. A. .. A multi-objective evolutionary method for Dynamic Airspace Resectorization using sectors clipping and similarities. Evolutionary Computation (CEC), 2012 IEEE Congress on. IEEE, (2012)

[5] Cao, L. , Xu, L. , Goodman, E. D. , \& Li, H. .. Decomposition-based evolutionary dynamic multiobjective optimization using a difference model. Applied Soft Computing, 76, 473-490, (2019).

[6] Bui, L. T. , \& Michalewicz, Z. .. An evolutionary multi-objective approach for dynamic mission planning. Proceedings of the IEEE Congress on Evolutionary Computation, CEC 2010, Barcelona, Spain, 18-23 July 2010. IEEE, (2010).

[7] Curtis, S. K. , Mattson, C. A. , \& B. J. Hancock..... Divergent exploration in design with a dynamic multiobjective optimization formulation. Structural \& Multidisciplinary Optimization, 47(5), 645-657, (2013). 
[8] Jun, H. , Liqian, X. , Cong-Cong, X. , \& Qiang, D. .. A novel bioinspired multiobjective optimization algorithm for designing wireless sensor networks in the internet of things. Journal of Sensors, 2015, 1-16, (2015).

[9] Macedo, Luís Lobato, Godinho, P. , \& Alves, M. J. .. Mean-semivariance portfolio optimization with multiobjective evolutionary algorithms and technical analysis rules. Expert Systems with Applications, 79, 33-43, (2017).

[10] Zhang, Z. .. Multiobjective optimization immune algorithm in dynamic environments and its application to greenhouse control. Applied Soft Computing Journal, 8(2), 959-971, (2008).

[11] Kim, K. , Mckay, R. , \& Moon, B. R. .. Multiobjective evolutionary algorithms for dynamic social network clustering. Genetic \& Evolutionary Computation Conference (pp.1179). DBLP, (2010).

[12] Linnala, M. .. Bi-level optimization for a dynamic multiobjective problem. Engineering Optimization, 44(2), 195-207, (2012).

[13] Fogel, L. J. , \& Owens, A. J. . Artificial intelligence through simulated evolution. Biophysics and Cybernetic Systems, (1966).

[14] Xu, H. , Wang, S. , \& Wu, S. Y. .. Optimization methods, theory and applications $\|$ on constraint qualifications for multiobjective optimization problems with vanishing constraints., 10.1007/978-3-662-47044-2(Chapter 6), 95-135, (2015).

[15] Yang, S., Nguyen, T. T. , \& Li, C. .. Evolutionary Dynamic Optimization: Test and Evaluation Environments. Evolutinary Computation for Dynamic Optimization Problems, (2013).

[16] Rong, M. , Gong, D. , Pedrycz, W. , \& Wang, L.... A Multi-Model Prediction Method for Dynamic Multi-Objective Evolutionary Optimization. IEEE Transactions on Evolutionary Computation, 24(2),290-304, (2019).

[17] A, A. Z. , B, B. Y. Q. , C, H. L. , B, S. Z. Z. , B, P. N. S. , \& D, Q. Z. .. Multiobjective evolutionary algorithms: a survey of the state of the art. Swarm and Evolutionary Computation, 1( 1), 32-49, (2011).

[18] Cheng, R. , Jin, Y. , Narukawa, K. , \& Sendhoff, B. .. A multiobjective evolutionary algorithm using gaussian processbased inverse modeling. IEEE Transactions on Evolutionary Computation, 19(6), 838-856, (2015).

[19] Deb K, Jain H. An evolutionary many-objective optimization algorithm using reference-point -based nondominated sorting approach, Part I: Solving problems with box constraints. IEEE Transactions on Evolutionary computation, 18(4), 577-601, (2014).

[20] Nguyen, T. T. , Yang, S. , \& Branke, J. .. Evolutionary dynamic optimization: a survey of the state of the art. Swarm \& Evolutionary Computation, 6, 1-24, (2012).

[21] Alyafeai, Z., Alshaibani, M. S. , \& Ahmad, I. .. A survey on transfer learning in natural language processing, (2020).

[22] Iason Hatzakis, D. W. .. Dynamic multi-objective optimization with evolutionary algorithms: a forward-looking approach. Conference on Genetic \& Evolutionary Computation. ACM, (2006).

[23] Christian von Lücken, Benjamín Barán, \& Brizuela, C. .. A survey on multi-objective evolutionary algorithms for many- 
objective problems. Computational Optimization \& Applications, 1-50, (2014).

[24] Zhou, A. , Jin, Y. , \& Zhang, Q. .. A population prediction strategy for evolutionary dynamic multiobjective optimization. IEEE Transactions on Cybernetics, 44(1), 40-53, (2013).

[25] Wei, J. , \& Zhang, M. .. Simplex Model Based Evolutionary Algorithm for Dynamic Multi-Objective Optimization. International Conference on Advances in Artificial Intelligence. Springer-Verlag, (2011).

[26] Min Liu.. Memory Enhanced Dynamic Multi-Objective Evolutionary Algorithm Based on Decomposition. Journal of software, 24(7): 1571 - 1588, (2014).

[27] Azzouz, R. .. A Multiple Reference Point-based evolutionary algorithm for dynamic multi-objective optimization with undetectable changes. Evolutionary Computation (CEC), 2014 IEEE Congress on. IEEE, (2014).

[28] Rethnaraj, Rambabu, Prahlad, Vadakkepat, Kay, \& Chen, et al.. A mixture-of-experts prediction framework for evolutionary dynamic multiobjective optimization. IEEE Transactions on Cybernetics, (2019).

[29] Mardé Greeff, \& Engelbrecht, A. P. .. Solving dynamic multi-objective problems with vector evaluated particle swarm optimisation. Evolutionary Computation. IEEE, (2019).

[30] Jiang, S. , \& Yang, S. .. A steady-state and generational evolutionary algorithm for dynamic multi-objective optimization. IEEE transactions on evolutionary computation: A publication of the IEEE Neural Networks Council, 21(1), 65-82, (2017).

[31] R. Liu, W. Zhang, L. Jiao, F. Liu, and J. Ma. A sphere-dominance based preference immune-inspired algorithm for dynamic multiobjective optimization. in Proc. GECCO, (2011).

[32] Jiang, S. , \& Yang, S. .. Evolutionary dynamic multiobjective optimization: benchmarks and algorithm comparisons. IEEE Transactions on Cybernetics, 198-211, (2016).

[33] Chun'An, L. , \& Yuping, W. .. Multiobjective evolutionary algorithm for dynamic nonlinear constrained optimization problems. Systems Engineering and Electronics, Journal of,20(1), p.204-210, (2009).

[34] Shouyong, Jiang, Shengxiang, \& Yang.. A steady-state and generational evolutionary algorithm for dynamic multiobjective optimization. IEEE Transactions on Evolutionary Computation, 21(1), 65-82, (2017).

[35] Ma, Y. , Liu, R. , \& Shang, R. .. A hybrid dynamic multi-objective immune optimization algorithm using prediction strategy and improved differential evolution crossover operator, (2011).

[36] Wei, J. , \& Wang, Y. .. Hyper rectangle search based particle swarm algorithm for dynamic constrained multi-objective optimization problems. Evolutionary Computation. IEEE, (2012).

[37] Yang, M. , Kang, L. , \& Guan, J. .. Multi-algorithm co-evolution strategy for Dynamic Multi-Objective TSP. IEEE Congress on Evolutionary Computation. IEEE, (2008).

[38] Wang, P. , \& Tong, X. .. A dimension convergence-based evolutionary algorithm for many-objective optimization problems. IEEE Access, 8, 224631-22464, (2020). 
[39] Azevedo, C. R. B. , \& Aluizio F. R. Araújo.. Generalized immigration schemes for dynamic evolutionary multiobjective optimization, (2011).

[40] Wang, Y. , \& Li, B. .. Investigation of memory-based multi-objective optimization evolutionary algorithm in dynamic environment. Evolutionary Computation. cec.ieee Congress on, 630 - 637, (2009).

[41] Mario Cámara, Ortega, J. , \& Toro, F. D. .. Approaching Dynamic Multi-Objective Optimization Problems by Using Parallel Evolutionary Algorithms. Advances in Multi-Objective Nature Inspired Computing. Springer Berlin Heidelberg, (2010).

[42] Vinek, E. , Beran, P. P. , \& Schikuta, E. .. A dynamic multi-objective optimization framework for selecting distributed deployments in a heterogeneous environment. Procedia Computer Science, 4(none), 166-175, (2011).

[43] Helbig, M. , \& Engelbrecht, A. P. .. Analyses of guide update approaches for vector evaluated particle swarm optimisation on dynamic multi-objective optimisation problems. 2012 IEEE Congress on Evolutionary Computation. IEEE, (2012).

[44] Mario Cámara, Ortega, J. , \& Toro, F. D. .. A single front genetic algorithm for parallel multi-objective optimization in dynamic environments. Neurocomputing, 72(16-18), 3570-3579, (2009).

[45] Li, Ke, Kwong, Sam, Deb, \& Kalyanmoy.. A dual-population paradigm for evolutionary multiobjective optimization. Information Sciences, (2015).

[46] Peng, Z. , Zheng, J. , \& Zou, J. .. A population diversity maintaining strategy based on dynamic environment evolutionary model for dynamic multiobjective optimization. Evolutionary Computation. IEEE, (2014).

[47] Gretton, A. , Borgwardt, K. M. , Rasch, M. J., Schlkopf, B. , \& Smola, A. J. .. A kernel method for the two-sampleproblem. Advances in neural information processing systems, 1, (2007).

[48] Jiang, M. , Huang, Z., Qiu, L. , Huang, W. , \& Yen, G. G. .. Transfer learning based dynamic multiobjective optimization algorithms, (2016).

[49] Smola, A. , Gretton, A. , Song, L. , \& Schoelkopf, B. .. A Hilbert Space Embedding for Distributions. International Conference on Discovery Science. Springer Berlin Heidelberg, (2007).

[50] C Raquel , Y Xin.. Dynamic Multi-objective Optimization: A Survey of the State-of-the-Art, (2013).

[51] Goh, C. K. , \& Tan, K. C. .. A competitive-cooperative coevolutionary paradigm for dynamic multiobjective optimization. IEEE Press, (2009).

[52] M. Helbig and A. Engelbrecht. Benchmark functions for CEC 2015 special session and competition on dynamic multiobjective optimization. Comput. Sci. Dept., Univ. Pretoria, Pretoria, South Africa, Tech. Rep., (2015).

[53] ZHENG Jin-hua, PENG Zhou, et al.. A Prediction Strategy Based on Guide-Individual for Dynamic Multi-Objective Optimization. ATA ELECTRONICA SINICA, 43(9), 1816-1825, (2015). 
Figures

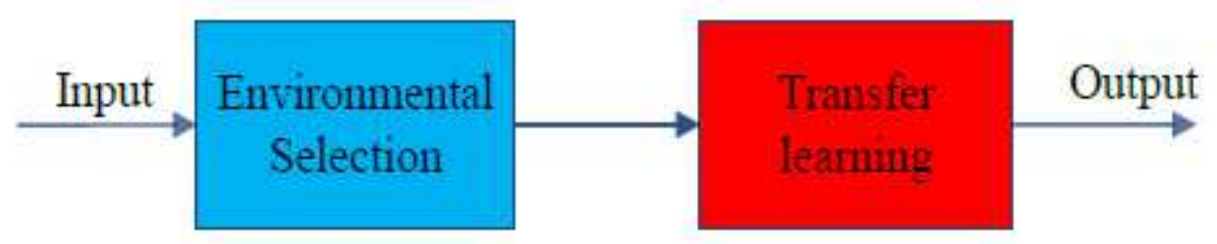

Figure 1

Principle of DMOEA-ESTL Strategy

\begin{tabular}{|c|c|c|c|c|c|}
\hline - A & & & & & $\leftarrow$ Environment Domain \\
\hline & - $\mathrm{B}$ & & & & \\
\hline & - $\mathrm{C}$ & - $\mathrm{D}$ & & & \\
\hline & & & - $\mathrm{E}$ & $\leftarrow$ & Unit Domain \\
\hline & & & & $\because \mathrm{F}$ & \\
\hline & & & & & - $\mathrm{H} \longleftarrow$ Individual \\
\hline
\end{tabular}

Figure 2

Individuals in Environment Domain and Unit Domain

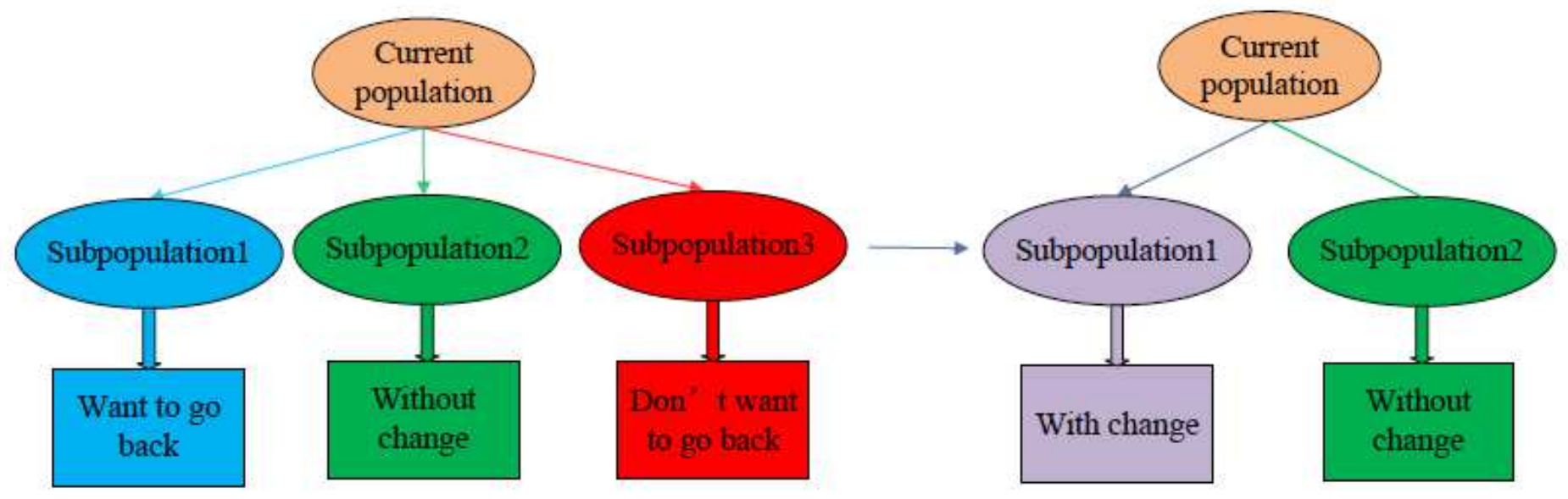

Figure 3

Division of Population 


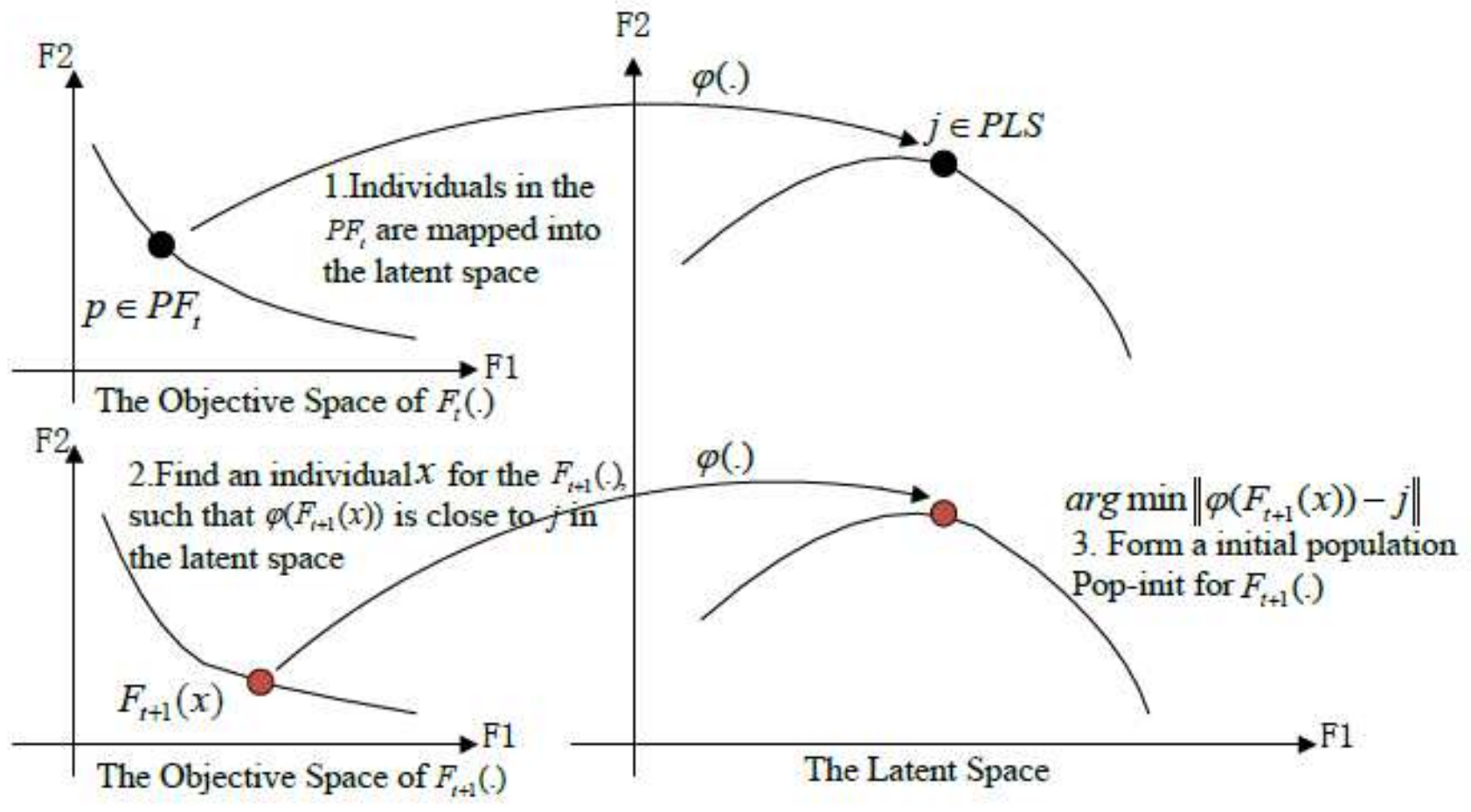

\section{Figure 4}

Key Steps of DMOEA-TL Algorithm. F1DF2 represent the objective function respectively.

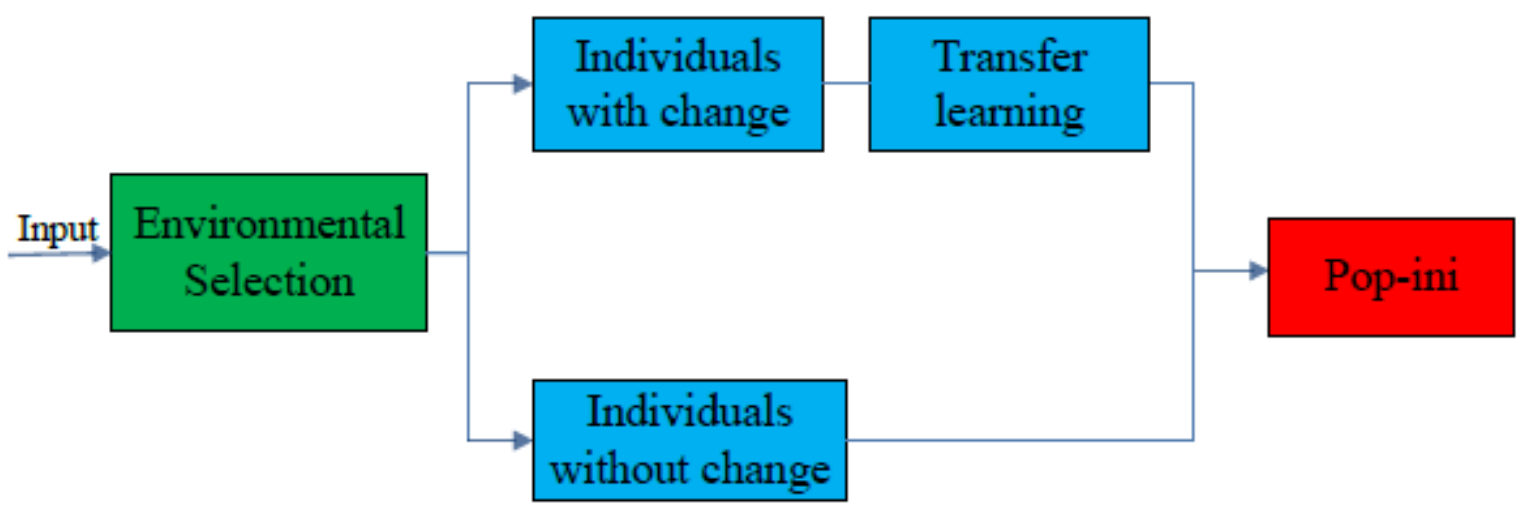

Figure 5

Principle of DMOEA-ESTL
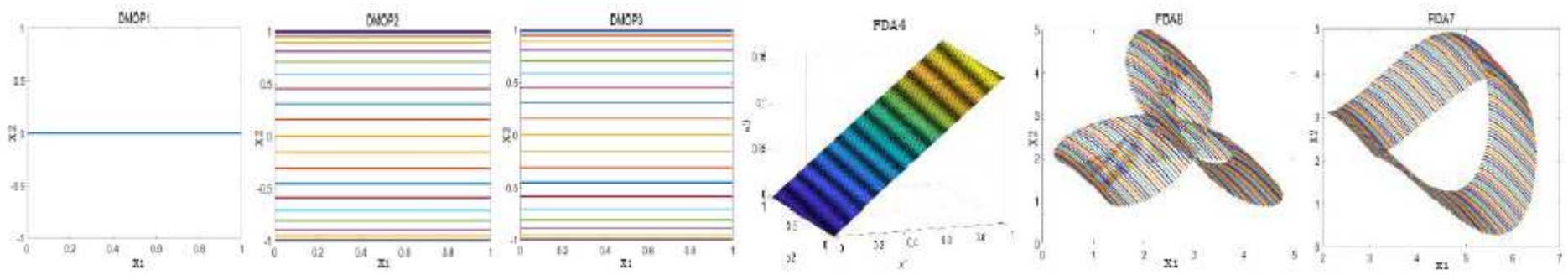

Figure 6 

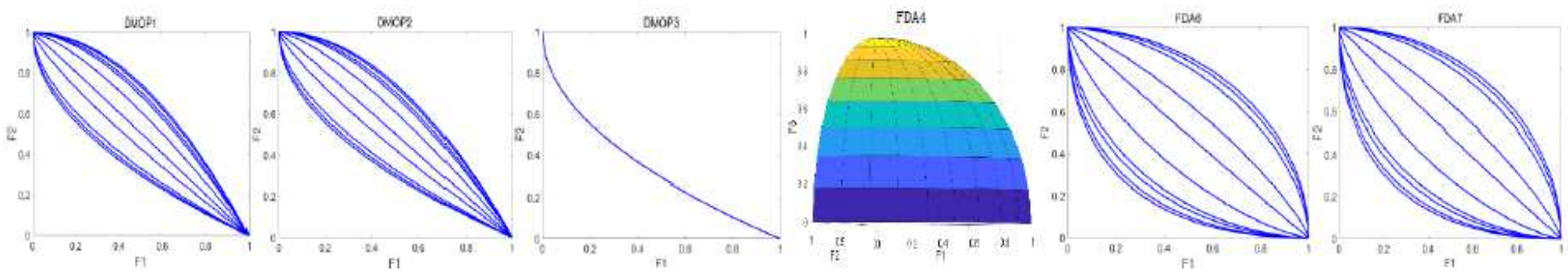

Figure 7

True PFs of the Six Testing Functions
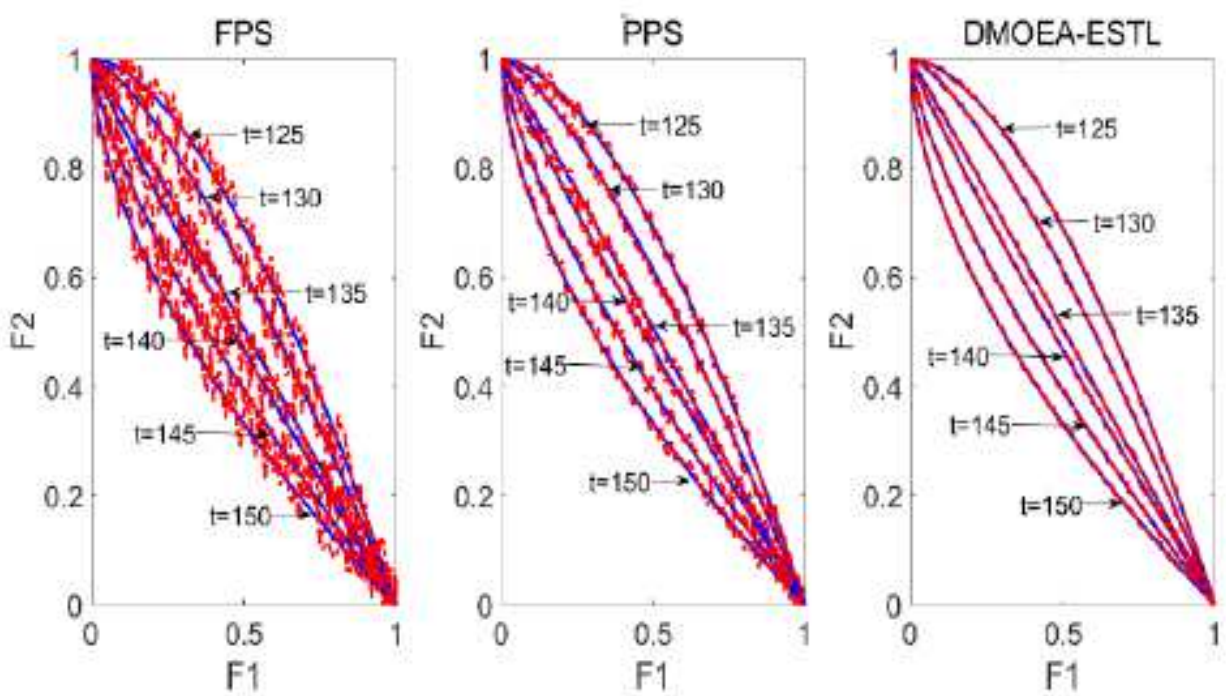

Figure 8

Populations Obtained by FPS, PPS, and DMOEA-ESTL on DMOP1 Figure
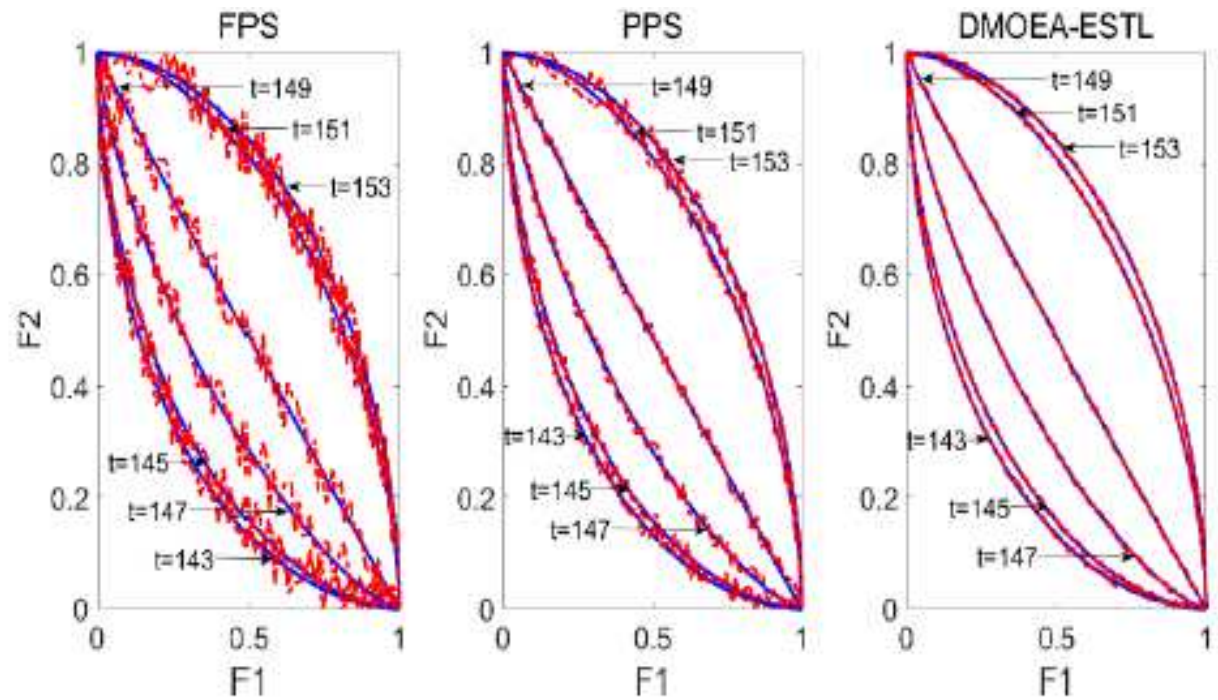

Figure 9 
Populations Obtained by FPS, PPS, and DMOEA-ESTL on FDA6
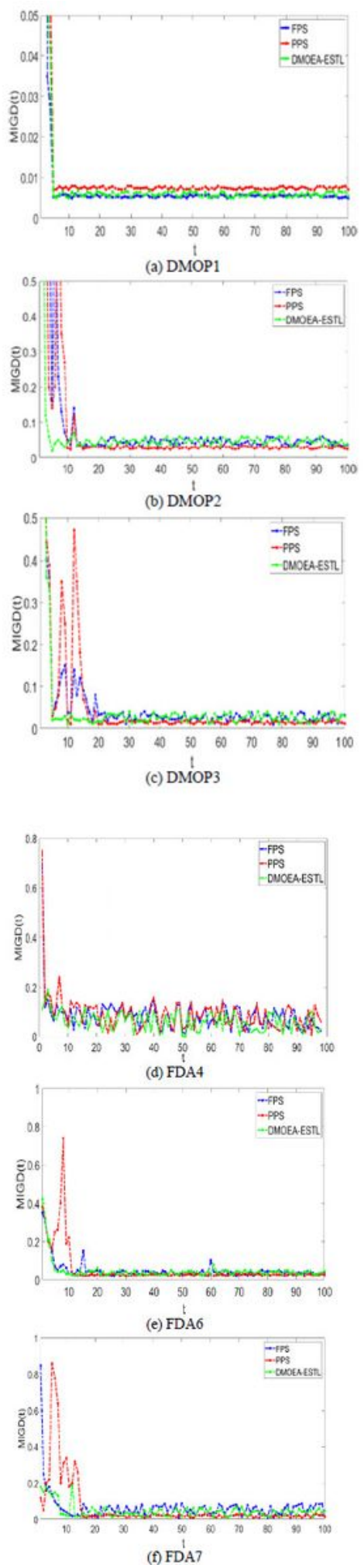

\section{Figure 10}

Average MIGD of Three Algorithms 